\title{
The Potential Benefits of Compressed Sensing and Machine Learning for Advanced Imaging and Spectroscopy in the Electron Microscope
}

\author{
Nigel Browning ${ }^{1}$, Robert Klie ${ }^{2}$, Anthony Barker ${ }^{1}$, Andrew Stevens ${ }^{1}$ and Christopher Buurma ${ }^{1}$ \\ ${ }^{1}$ Sivananthan Laboratories, Inc., Bolingbrook, Illinois, United States, ${ }^{2}$ University of Illinois at Chicago, \\ Chicago, Illinois, United States
}

While Machine Learning (ML) has made numerous advances in the analysis of "big data", as this type of data is initially generated for other purposes, ML tends to uncover new insights by serendipity, i.e. valuable previously unknown trends in the data are found, but which specific areas these trends will relate to, cannot be determined a priori. Scientific data used to characterize materials or processes on the other hand is generated with a specific project in mind from highly specialized and expensive equipment, making the generation and analysis of a diverse "big dataset" impossible. However, we can use the way that ML analyses "big data" to learn which parts of any given dataset are most useful for interpretation, and then design our experiments to generate only that "targeted data" which is most useful. For state-ofthe-art electron microscopy, compressively sensing (CS) and reconstructing images / spectra using an ML framework is particularly valuable for obtaining quantitative multi-scale hyperspectral data under extremely low dose and/or dose rate conditions with significantly accelerated framerates. The dose / speed / resolution optimization that is possible using these methods creates wide-ranging new opportunities to avoid major electron beam damage and enables quantified observations of metastable materials and materials dynamics to be made.

The benefits of CS/ML for electron microscopy can be easily understood if we consider the types of datasets that can be obtained in a microscope. In SEM, TEM and STEM, 2D (with $x$ and y spatial coordinates for each pixel), 3D (x, y, z) and hyperspectral (x, y, z, DE) images all record an intensity, I (with a number of independent grey scales), at each pixel at each particular time interval Dt. In some forms of images, such as ptychography and electron backscattered diffraction (EBSD), the intensity itself can have a 2D distribution, I (x, y) - a complete 2D image/diffraction pattern is acquired at each 3D location. For a complete $3 \mathrm{D}$, dynamic hyperspectral ptychographic image there would be 7 dimensions to the dataset, that with a standard resolution of 1,000 data points (pixels) per dimension would have $10^{21}$ total pixels! Recording such a dataset would obviously be impossible under standard image acquisition conditions, even if each pixel requires only a picosecond. If we could decrease the number of measurements being made in each dimension, however, we could significantly reduce the time and electron dose on the sample needed to achieve this resolution. As an example of how this could work, Figure 1 shows the example of the reconstruction of a hyperspectral set of images from a satellite, where only $0.5 \%$ of the data is needed to accurately reproduce the full dataset. A similar result is shown in Figure 2 , with a simulated atomic resolution $\mathrm{Z}$-contrast image as the test case.

The key to using this approach successfully is knowing how many of the pixels you can eliminate in each dimension and still be able to extract the information that you want from the image (and it is not clear that each dimension should have the same number of pixels). Additionally, for the imaging approach to have wide-ranging applications, it must be able to adapt to the sample, scene, or process being imaged, always using the optimum number of pixels required to achieve the spatial, temporal and spectroscopic resolution needed to characterise the results uniquely. A combined hardware/software system to achieve this flexible and optimal sampling approach is currently being developed by Sivananthan Laboratories and is being tested on Z-contrast, ABF, ptychography, EELS and EDS in the STEM. In this presentation, we discuss 
the progress with these methods and limitations to these methods imposed by their practical implementation on existing microscopes [1-6].

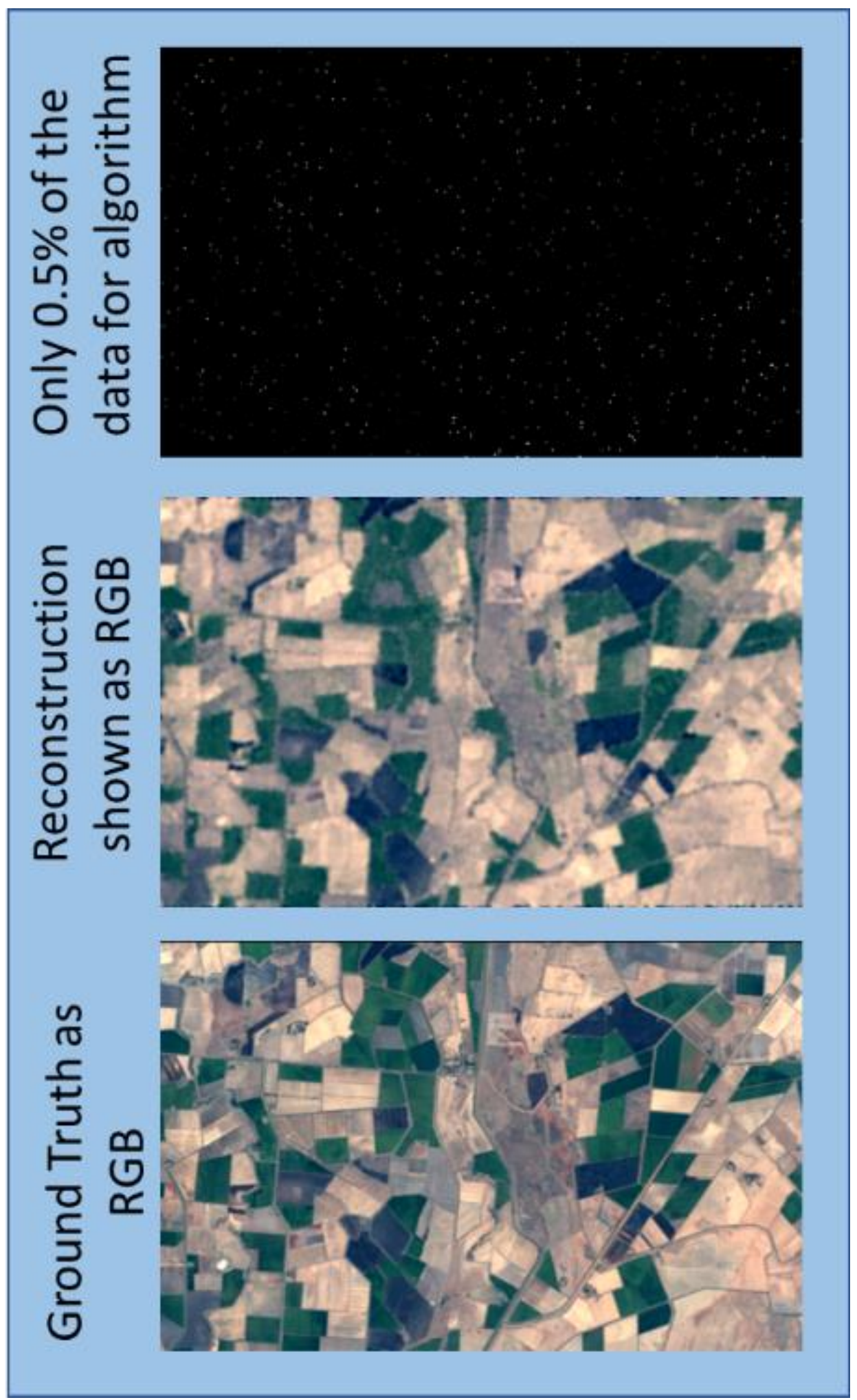


Figure 1. (left) A fully sampled image can be recovered (center) using $0.5 \%$ of the pixels and an inpainting algorithm (right).
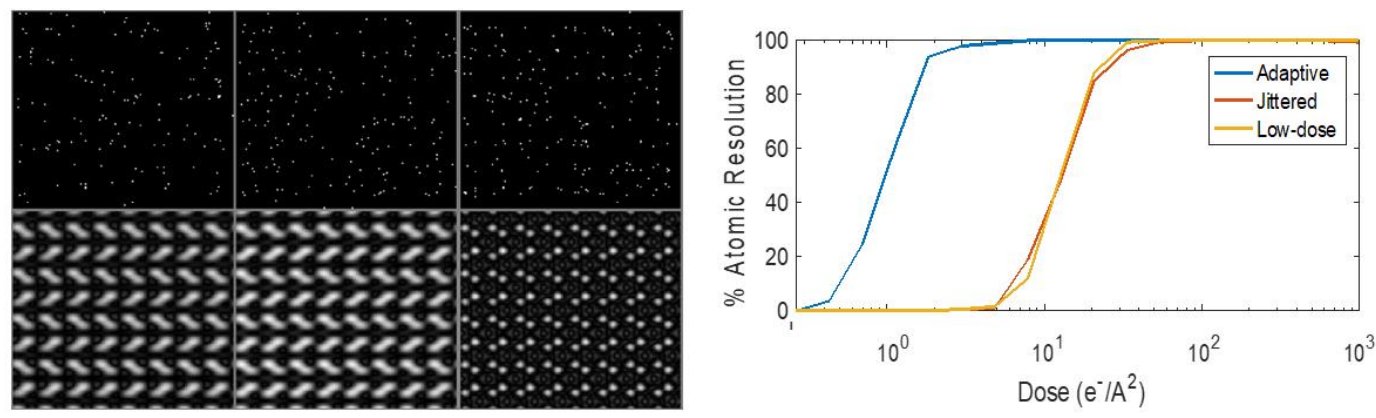

Figure 2. Representative reconstructions for Z-contrast images with a dose of 10 e-/Å2. (a) Low-dose, (b) Jittered and (c) adaptive reconstructions from the sub-sampled images. The jittered/adaptive reconstructions use $10 \%$ of the pixel locations. (d) the resolution of the low-dose and jittered images is the same but jittered is 10x faster. Adaptive sensing is 10x faster and the same resolution can be obtained with 10x less dose.

\section{References}

1. Stevens A., Yang H., Carin L., Arslan I., and Browning N. D. (2014) The potential for Bayesian compressive sensing to significantly reduce electron dose in high-resolution STEM images. Microscopy 63, (1) 41-51.

2. Stevens A., Kovarik L., Abellan P., Yuan X., Carin L., and Browning N. D. (2015) Applying compressive sensing to TEM video: A substantial frame rate increase on any camera. Advanced Structural and Chemical Imaging 1, (1) 1-20.

3. Kovarik L., Stevens A., Liyu A., Browning N. D (2016) Implementing an Accurate and Rapid Sparse Sampling Approach for Low-Dose Atomic Resolution STEM Imaging of Electron Beam Sensitive Materials", APL 109, 164102

4. Stevens A., Yang H., Luzzi L., Kovarik L., Mehdi B. L., Liyu A., Gehm M. E., Browning N. D. (2017) Sub-sampled Approaches for Extremely Low-Dose STEM, APL112, 043104 (2018)

5. Stevens A., Yang H., Hao, W., Jones, L., Ophus, C., Nellist, P. D., Browning N. D. (2018) Sub-sampled STEM-Ptychography, APL 113, 033104

6. Mehdi B. L., Stevens A., Kovarik L., Jiang, N., Mehta, H., Liyu, A., Reehl, S., Stanfill, B., Luzzi, L., MacPhee, K., Bramer, L., Browning, N. D. (2019) Controlling and Observing the Kinetics of Nucleation and Growth by Sub-Sampling Dynamic In-Situ STEM Images, APL 115, 063102 